\title{
Strates
}

STRATES Matériaux pour la recherche en sciences sociales

$4 \mid 1989$

Dossier : images réfléchies. Paroles d'un paysan révolutionnaire

\section{Espace graphique et pratiques de pouvoir : philosophies d'entreprises à Taïwan, préceptes politiques à Singapour}

\section{Catherine Paix et Michèle Petit}

\section{OpenEdition}

\section{Édition électronique}

URL : http://journals.openedition.org/strates/4262

DOI : $10.4000 /$ strates.4262

ISSN : 1777-5442

\section{Éditeur}

Laboratoire Ladyss

Édition imprimée

Date de publication : 31 décembre 1989

ISSN : 0768-8067

\section{Référence électronique}

Catherine Paix et Michèle Petit, « Espace graphique et pratiques de pouvoir : philosophies

d'entreprises à Taïwan, préceptes politiques à Singapour », Strates [En ligne], 4| 1989, mis en ligne le

19 mai 2008, consulté le 08 septembre 2020. URL : http://journals.openedition.org/strates/4262 ;

DOI : https://doi.org/10.4000/strates.4262

Ce document a été généré automatiquement le 8 septembre 2020.

Tous droits réservés 


\title{
Espace graphique et pratiques de pouvoir : philosophies d'entreprises à Taïwan, préceptes politiques à Singapour
}

\author{
Catherine Paix et Michèle Petit
}

« Exprimer, ou plutôt figurer, ce n'est point simplement évoquer, mais susciter, mais réaliser (...) Le bon ordre dépend entièrement de la correction du langage ». Marcel GRANET, « La pensée chinoise »"

«Si les désignations ne sont pas correctes, les paroles ne peuvent être conformes, si les paroles ne sont point conformes, les affaires n'ont aucun

succès ».

TSEU-LOU à CONFUCIUS

1 Tout homme soucieux d'avoir prise sur ses semblables, sans être Chinois, paraît saisir instantanément la fonction "mandarinale» de l'écriture. Mais en pays chinois, il semble que dans le tri qu'opère la mémoire, le souvenir de l'efficience attribuée aux mots écrits, et du pouvoir que leur maniement procure pour unifier et guider l'action des hommes, soit restée particulièrement vivace.

Beaucoup plus concrète que les langues occidentales, du fait sans doute de l'origine pictographique des idéogrammes, la langue chinoise est aussi plus vivante, en ce sens où les mots incarnent des représentations imagées, évocatrices de qualités, de relations ou d'actions inscrites dans des réalités singulières qui lui confèrent - plus qu'à toute autre - la faculté de suggérer l'action et de la provoquer en la figurant.

Ce caractère éminemment "emblématique $»^{2}$ de la langue aide à mieux comprendre que l'écriture ait été un instrument majeur du pouvoir politique en Chine, les mots ayant de ce fait la force quasi-magique de maintenir les êtres et les choses à la place qui devait être la leur dans l'Ordre social établi. Dans la Chine ancienne, rendre les 
désignations correctes et fournir à ceux qu'il dirigeait les emblèmes et devises étaient la première obligation du « Prince », ce qui lui permettait de fixer et d'imposer règles et hiérarchie sociale. Acquis au terme d'une longue initiation, le chinois littéraire était, dans la Chine impériale, le privilège d'une élite, la langue des maîtres, le ciment de l'Empire. Véritable « espéranto pour les yeux »', il pouvait être lu partout, alors même que les prononciations extrêmement variables interdisaient souvent de se comprendre à quelques kilomètres de distance. Et ce sont les fonctionnaires lettrés, qui détenaient le pouvoir et se situaient au plus haut de la hiérarchie confucéenne, qui ont assuré tout au long des siècles l'unité politique et culturelle.

4 Aujourd'hui, le recours au symbolique et tout particulièrement aux mots écrits - quand ceux-ci ne font pas loi - semble garder une place majeure dans l'art de gouverner en pays chinois, et on ne peut manquer d'être frappé par l'importance que lui accordent ceux qui y détiennent le pouvoir économique et politique. Il en est ainsi à Taïwan et à Singapour, où les signes linguistiques ou iconiques qu'ils produisent semblent plus chargés d'efficacité pratique que les logos et les « cultures » d'entreprises ou les slogans politiques développés en Occident, plus directement attachés à amener chacun à s'y soumettre, comme s'ils avaient le pouvoir de guider l'action.

5 A Taïwan, les entrepreneurs, des plus traditionnels aux plus modernes, attirent tous l'attention du visiteur sur leurs "philosophies ", condensées en devises - tracées au pinceau, intaillées dans le bronze, ou simplement imprimées, selon les graphies traditionnelles en usage ${ }^{4}$ - qui ornent, tels des dazibao, bureaux et usines, ou qui enluminent les brochures de présentation de leurs sociétés, quand ils ne s'empressent pas de se faire photographier devant ${ }^{5}$. Et, dans tous les cas, leurs auteurs accordent une grande efficience à ces principes, qu'ils ont posés comme acte fondateur de leur entreprise, et les placent au premier rang de leur réussite. (cf. Doc. en annexe. Un entrepreneur de Taïwan et ses préceptes.)

6 A Singapour, c'est l'espace des rues et le temps d'une journée qui se trouvent rythmés par les prescriptions omniprésentes du pouvoir politique, sous forme d'affiches, de spots télévisés et de slogans dont les termes moralisateurs sont censés s'immiscer au cœur de chacun et l'impliquer concrètement dans la mise en œuvre des directives gouvernementales.

7 Marques de souveraineté sur un territoire, productrices d'identité pour un territoire, ces « écritures exposées " ${ }^{6}$, en raison même de l'importance que semblent leur conférer leurs auteurs dans la mise en œuvre de leurs stratégies économiques et politiques, et de l'ampleur des enjeux liés à leur maniement, nous sont apparues ainsi comme un chemin de traverse pour mieux comprendre les conceptions et pratiques du pouvoir dans ces pays.

Taïwan, plus que donner une éthique à l'entreprise : assumer une fonction sociale et politique.

8 L'importance qu'attachent les entrepreneurs de Taïwan à édicter sous forme de maximes les principales valeurs morales et les attitudes qu'ils estiment indispensables à la bonne marche de leur entreprise est irréductible à la seule logique du profit, et ne peut être mise sur le compte d'une rationalité économique qui serait indépendante de leur culture et de leur histoire ${ }^{7}$. Elle atteste, au contraire, une conception très particulière des rapports sociaux au sein de l'entreprise - et notamment du rôle du leader -. Et elle relève étroitement d'un souci de reconnaissance sociale et politique, 
voire d'indépendance à l'égard du pouvoir, et de la volonté d'affirmer une identité au plan international, qui sont tout à fait propres aux entrepreneurs de ce pays.

Ce que ceux-ci appellent leurs "philosophies", et qui consiste, à partir de concepts riches de significations, à produire une vision synthétique de la firme en exprimant sous forme idéalisée la façon de s'y comporter et d'y travailler, est indissociable du modèle de relations familiales et communautaires dans lequel se trouvent enracinées les entreprises chinoises.

10 Dans le fait d'énoncer ces concepts, il y a en effet le souci de professer qui témoigne d'une certaine transposition au sein de l'entreprise de la fonction didactique traditionnelle du père au sein de sa famille et du pouvoir, comme du prestige, que cette fonction lui confère parmi les siens ; comme lui, le patron, censé travailler pour le bien du groupe, se considère comme son guide moral et spirituel, celui qui indique le sens à donner à l'activité, fixe les objectifs, et enseigne les moyens d'y parvenir.

11 Encore maintenant, la famille demeure au cœur des entreprises de Taïwan. Et dans certaines d'entre elles, où l'autorité incontestée du chef d'entreprise et la soumission à ses directives cèdent le pas à une responsabilisation du personnel aux différents niveaux de la hiérarchie, les dirigeants s'appuient toujours dans leurs discours sur ces maximes pour expliquer et justifier dans quelles directions ils ont estimé nécessaire d'orienter l'investissement, ou pour impulser de nouvelles formes d'organisation du travail, et susciter l'engagement des salariés et des actionnaires au devenir de la firme.

12 La création de Fondations à vocation sociale et culturelle, et d'Instituts de formation, auxquels ils attachent la plus haute importance, n'ont pas non plus pour seule fonction de former une main-d'œuvre qualifiée ${ }^{8}:$ il s'agit aussi dans ce cadre d'inculquer aux candidats au recrutement l'éthique de l'entreprise, en montrant en quoi celle-ci a été le gage de sa réussite, et d'en transmettre une représentation qui aura pour eux valeur d'identité et d'idéal, et les stabilisera dans l'entreprise, comme membres d'une communauté soudée aux objectifs de ses dirigeants. Certaines firmes n'organisent-elles pas des travaux pratiques pour montrer aux employés ce que la production à laquelle ils ont contribué peut apporter de progrès dans leur vie quotidienne, voire plus largement à la société ! Et parmi la nouvelle génération d'entrepreneurs de Taïwan, qui n'ont pas démarré leurs affaires sur des bases familiales, la formation du personnel, la diffusion des compétences et des ambitions des dirigeants, comme vecteur d'identification des salariés, constituent le pivot de la «culture » de l'entreprise: ce qui contribue, en attachant les employés qualifiés à la firme, à développer ses capacités d'innovation et sa compétitivité sur le marché international.

Cette valorisation de la fonction éducative du leader est une donnée commune aux entreprises chinoises et japonaises et elle a été - en raison même de son efficience économique - largement reprise par les gestionnaires américains de l'« excellence ${ }^{9}$. Et à Taïwan comme ailleurs, elle est bien évidemment liée aux avantages qu'elle confère en termes de contrôle, de productivité, de négation des conflits, et aussi d'adaptation aux mutations technologiques et au marché international.

Mais le fait d'énoncer sous forme de préceptes leur philosophie semble être une pratique à laquelle sont tout particulièrement attachés les entrepreneurs de Taïwan ; on ne la retrouve pas, par exemple, dans les entreprises chinoises de Singapour, au sein desquelles les formes de gestion - toujours très marquées par les règles familiales - ne sont pourtant pas très différentes. L'importance qu'ils accordent au symbolique est indissociable de la position très inconfortable dans laquelle ils se sont trouvés placés 
dans la période qui a suivi le repli du gouvernement nationaliste dans l'île en 1949 ; elle renvoie aussi au rôle qui leur a été conféré par le régime pour asseoir son pouvoir et l'assurer d'une légitimité au plan national et international.

Soutenus par le gouvernement, les entrepreneurs originaires de Chine continentale ${ }^{10}$ ont eu à faire face à la méfiance ou à l'animosité de la population locale, et ont cherché à légitimer leur présence et à s'affirmer dans la société taïwanaise, en prouvant leurs capacités d'entrepreneurs industriels, voire en affichant une probité morale.

Issus de l'élite locale qui se trouva contrainte par le régime à limiter ses ambitions à la libre entreprise en raison de l'accaparement de l'administration et du pouvoir politique par les Chinois du continent, les entrepreneurs taïwanais ont cherché pour leur part à consolider leur position économique pour s'imposer.

Ayant tous en mémoire la conception très étatiste du gouvernement nationaliste en Chine, et obligés pour la plupart à réactiver les réseaux de solidarité familiale et communautaire pour financer leurs affaires, ils se sont attachés - sans doute plus qu'ailleurs - à les doter d'une autonomie financière et d'une forte cohésion sociale.

Une façon pour eux de résister aux aléas des décisions gouvernementales, et d'éviter la tendance à l'éclatement et à la dispersion qui était à l'époque inhérente aux entreprises chinoises.

S'imposant de l'extérieur, coupé de la population locale - qui gardait l'amer souvenir des affrontements de 1947, au cours desquels l'armée nationaliste s'était livrée à l'élimination physique de l'élite taïwanaise - le gouvernement a cherché, lui, à légitimer son pouvoir et à le consolider par la voie de la "réussite » économique, et il a donné dans ce cadre les possibilités d'un développement du secteur privé, pour se doter d'une assise sociale et politique. Puis, lorsqu'il s'est trouvé en perte de légitimité sur le plan international, au moment où les grandes puissances ont reconnu la Chine Populaire, c'est aux entrepreneurs qu'il a confié la tâche de prendre le relais des diplomates pour le représenter dans l'arène internationale. L'entreprise devenait le symbole du régime, de ses capacités à développer le pays et à consolider sa position au sein de l'économie mondiale.

L'analyse de la production symbolique de trois types d'entreprises de Taïwan, et de l'utilisation qui en a été faite dans le cadre de leur développement, montre ainsi que l'attention maintenue des entrepreneurs de ce pays à énoncer des préceptes ayant valeur de philosophie traduit largement le souci d'assumer une fonction sociale et politique et que cela n'a pas été sans jouer un rôle important dans leur "réussite " économique, et leurs capacités à s'imposer.

TATUNG : «Ainsi le monde sera une famille »

Chez TATUNG, la «nouvelle culture " qu'il s'agit d'édifier, si l'on en croit la chansonmaison, s'étaye sur une base solide et ancienne. L'une des plus vieilles entreprises de l'île - créée en 1918, c'était une des très rares sociétés taïwanaises d'envergure sous l'occupation japonaise -, TATUNG est aussi l'une des plus importantes, avec un chiffre d'affaires d'1,2 milliard de dollars US en 1987. Entreprise de travaux publics à l'origine, elle réalise bientôt une intégration dans la métallurgie, puis, après la seconde guerre mondiale, équipe les foyers taïwanais en appareils électroménagers. C'est surtout à partir des années soixante que la firme a connu une croissance rapide et opéré une importante diversification horizontale, selon un schéma classique pour de nombreuses entreprises chinoises. Elle a développé de nombreuses productions électroniques - dont 
récemment la fabrication de périphériques informatiques - dont l'importance la situe au premier rang des producteurs locaux dans cette branche. Une part croissante de ces productions est vendue sur les marchés extérieurs, $35 \%$ du chiffre d'affaires étant aujourd'hui réalisé à l'exportation, ce qui place la firme parmi les tous premiers exportateurs du pays.

Aux 25000 salariés du groupe, il faudrait pouvoir ajouter - impossible à apprécier - la main d'œuvre mise au travail dans le cadre d'un système de sous-traitance très étendu.

"TATUNG " signifie « coopération fraternelle " : l'entreprise se place ainsi, dans son nom même, sous l'obédience de Confucius : "aide les autres comme toi-même", précise T.S. LIN $^{11}$, le fils du fondateur, à la tête du groupe depuis 1942, «pense aux autres comme à toi-même, et ainsi le monde sera une famille ». Etendre à la firme et à la nation, au « monde », la loyauté traditionnelle familiale, tranférer les valeurs familiales dans d'autres cadres, tel a donc été l'enjeu majeur pour le fondateur ${ }^{12}$. (cf. Doc. en annexe. Integration of management and labor autonomous management.)

Le "fraternalisme » cache évidemment le poids du " père ", et si les cadres doivent ici prendre soin « jour et nuit » des employés comme de leur famille, les relations patron/ employés sont du même coup inscrites sous le signe de la piété filiale et des vertus de soumission et de dévouement qui lui sont attachées.

C'est donc une philosophie à usage essentiellement interne qui est, ici, affichée : l'« esprit » de l'entreprise s'adresse aux membres de la famille, et les vertus exaltées "Intégrité, Honnêteté, Application, Frugalité " - qualifient toutes l'attitude requise des employés à l'intérieur de la firme, leur disposition envers le travail. Elles trouvent leur origine dans les valeurs paysannes, selon T.S. LIN, qui aime rappeler que ses ancêtres étaient agriculteurs. Plusieurs de ces vertus reviennent sous le pinceau d'autres entrepreneurs, d'origine taïwanaise, ou quelquefois continentale. On y décèle l'empreinte de cette tradition rurale, si prégnante en Chine Populaire comme en "Chine nationaliste». Et aussi le fait que c'est sur le monde rural que l'activité manufacturière s'est appuyée - dans le cadre d'une politique d'intégration de l'industrie et de l'agriculture - et que les campagnes sont encore l'un des ressorts essentiels de la production, par le biais de la sous-traitance .

Parmi ces vertus paysannes, la « frugalité » est souvent inscrite en lettres... d'or dans bien des entreprises. Elle a joué un rôle essentiel dans l'ascension rapide des entrepreneurs, en leur permettant non seulement de légitimer des politiques de bas salaires, mais aussi de faire appel, de façon déterminante, à l'épargne des travailleurs. Taïwan est l'une des sociétés les plus épargnantes du monde ${ }^{13}$, et la participation de l'épargne, consentie ou forcée, des employés et de leurs familles au développement de la firme est souvent un élément clé de leur développement, dans un pays où un système financier très rigide, pratiquement entièrement contrôlé par l'Etat, a toujours rendu très malaisée l'obtention de fonds.

Membres d'une même famille, les 25000 « collègues » de chez TATUNG - on n'y parle jamais de salariés - sont presque tous actionnaires de la société et possèdent ensemble près du tiers des actions. Cette application du principe «Intégration de la gestion et du travail ", encouragée par le Guomindang ${ }^{14}$, y a été développée dès 1945 . Au cours des années soixante et soixante-dix, l'emprunt auprès des employés et des actionnaires s'est systématisé, alors que les possibilités d'obtenir d'autres financements étaient 
restreintes, et les procédures très lourdes. Ces emprunts ont couvert alors les troisquarts des augmentations du fonds de roulement.

C'est au syndicat, évidemment "maison", qu'a été dévolue la tâche d'appliquer le principe "A chaque employé sa part », et de convertir les salariés en actionnaires, les actions étant cédées moyennant des prêts sans intérêt à long terme. Lui incombe aussi d'appliquer - d'une façon beaucoup plus sélective, puisqu'il ne concerne que 1500 d'entre eux - le principe "A chaque employé son logement ", par l'octroi de prêts sans intérêts.

Tout en renforçant l'identification à la firme, en impliquant toujours plus les employés dans son destin, ce drainage de leur épargne, et de celle de leurs proches, a ainsi permis au groupe de réaliser, tout au long des années, les augmentations de capital nécessaires à son expansion. Mais il lui a aussi permis d'agir, de fait, comme un banquier vis-à-vis d'autres entreprises, à des taux très supérieurs aux taux officiels, ce qui permettait de pallier les rigidités de l'appareil bancaire.

C'est toujours en « comptant sur ses propres forces » que la firme a défini sa politique de recrutement : plutôt que de faire appel à des compétences extérieures, elle a cherché à avoir son propre vivier - quand bien même le manque de main d'œuvre expérimentée viendrait à menacer telle ou telle reconversion de l'entreprise. Dès 1943, une Ecole Professionnelle fut fondée, bientôt suivie d'un Institut de Technologie. Dans la tradition confucéenne, selon laquelle diriger c'est éduquer, l'une et l'autre ont pour double fonction d'assurer la formation d'une main d'œuvre qualifiée, dans tous les champs d'activité de l'entreprise, et de diffuser la philosophie maison: aux enseignements techniques viennent ainsi s'adjoindre quelques cours de "philosophie morale", d'histoire chinoise, et l'étude des pensées de SUN Yat-sen. Les élèves sont censés apprendre à cultiver "l'autonomie technologique et l'indépendance académique » (la nuance est d'importance car en matière technologique, TATUNG ne semble pas prétendre à plus qu'à une autonomie...). L'éducation s'y veut concentrée sur la formation de "l'individu complet», assurant le "développement du caractère, une vision large, une sensibilité culturelle et une profonde sollicitude pour le genre humain ».

31 Ainsi instruits du sens de leur travail futur pour le bien être de l'humanité, les élèves, solidement encadrés - 200 enseignants, plus des consultants étrangers, pour 1000 élèves - apprennent aussi, vertu cardinale, «l'autoconduite": tout employé, tout cadre est invité à être son propre contremaître, en évaluant son travail et en le révisant dans le sens d'un meilleur rendement. Version appliquée de l'autocontrôlé confucéen...

Le cursus de plusieurs formations dispense enfin aux élèves un entraînement militaire, et l'entreprise dans son ensemble présente un style fortement militariste: c'est au garde-à-vous, et en claquant des talons, qu'on y accueille le visiteur. De tous temps, l'entreprise a d'ailleurs été dans les meilleurs termes avec les régimes militaires qui se sont succédés à la tête du pays : bon collaborateur, le fondateur de TATUNG a réalisé des travaux de construction et d'ingénierie pour le compte du colonisateur japonais, et participé à son effort d'armement; au lendemain de la guerre, l'entreprise répare les chemins de fer endommagés et, rallié activement au régime nationaliste, T.S. LIN deviendra membre du Comité Central du Guomindang, Président du Conseil de la ville de Taipeh, Président de la Fédération Nationale de l'Industrie - c'est l'un des très rares Taïwanais à avoir accédé, dès les années soixante, à des fonctions politiques importantes, alors monopolisées par les Chinois venus du continent lors de la débâcle de 1949. 

une large mesure la clé de l'accumulation, tant par le biais de commandes publiques, de l'accès au marché, extérieur ou intérieur, de l'obtention d'aides américaines..., que par l'information constante sur les grandes orientations à venir. En retour, le patron, homme de discipline, fait assumer à la firme une fonction sociale, clairement affichée dans ses mots d'ordre (" créer une nouvelle société ", « une nouvelle culture »), qui ne sont pas pur discours. Par exemple, si beaucoup des étudiants formés dans l'Institut travaillent ensuite dans la firme, la plupart d'entre eux, notamment dans certaines disciplines (comme l'administration et la gestion des affaires) sont employés dans d'autres entreprises, ou dans la fonction publique. Comme pour le financement, le « rayonnement » de TATUNG et de son « esprit » excède donc le territoire de la firme. On peut aussi voir s'esquisser, avec des entreprises comme TATUNG, une forme de répartition des rôles, où l'entreprise produit un véritable modèle pour la société dans son ensemble et où l'Etat autoritaire est déchargé de la fonction d'encadrement et de mise au pas de la population - alors que ce contrôle est totalement assumé par l'Etat, on le verra, à Singapour.

De la capacité effective de la firme à nouer des alliances avec des partenaires étrangers, et notamment des grands de l'électronique, rien ne transparaît dans les maîtres-mots de la philosophie. Et cela indique peut-être son point de fragilité. Autofinancement, autoconduite, autocontrôle, lui ont assuré "une solide base de pierre", comme dit l'hymne de l'Institut. Mais c'est dans son rapport aux autres et au monde extérieur que la «famille» semble la plus fragile. Les clients de TATUNG ne sont plus seulement les foyers taïwanais. Et si la firme a été l'une des premières à s'implanter en Asie, si elle a réalisé une douzaine d'implantations aux USA et en Europe, celles-ci la laissent pourtant loin derrière les groupes coréens. Son accès aux marchés est encore presque entièrement réalisé dans le cadre des accords «OEM $»^{15}$ qu'elle passe avec de grosses firmes américaines ou nippones, pour la plupart de ses productions en électronique. TATUNG n'a pu imposer sa propre marque de micro-ordinateurs, n'ayant pas conçu un produit qui soit compatible avec IBM ou avec APPLE. «Pense aux autres comme à toimême» ?... Il serait sans doute temps, pour cette firme, de penser aux autres comme différents d'elle-même...

Faible affirmation extérieure en son nom propre, recherche et développement insuffisamment développés, gestion bureaucratique, lente à réagir, diversification horizontale sur un trop grand nombre de produits, népotisme, manque de délégation effective des responsabilités: des faiblesses «classiques" de l'entreprise chinoise. L'ancrage dans la solidarité et la discipline familiale a largement fait la force de TATUNG. Mais aujourd'hui, cette tradition, un peu statufiée, à l'image du bronze du fondateur dont le regard pèse sur les générations suivantes, entrave la firme. Et c'est de l'extérieur, notamment des banques étrangères, que des pressions s'exercent pour que la gestion soit modernisée.

FAR EASTERN TEXTILE CORPORATION : S'imposer comme firme nationale au sein de l'espace économique mondial

Créée en 1952 par un industriel de Shanghaï ayant fui la Chine communiste en 1949 pour Hong-Kong, puis pour Taïwan quelques années plus tard, quand le gouvernement nationaliste manifesta sa volonté de donner aux entrepreneurs émigrés de Chine continentale les moyens de reconstituer un capital en reconnaissance de leur allégeance au Régime, la Compagnie FAR EASTERN se situe aujourd'hui au premier rang 
des producteurs et exportateurs de textiles de Taïwan. Diversifié dans d'autres branches d'activité, c'est aussi le deuxième groupe privé du pays, avec un chiffre d'affaires de 2 milliards de dollars US en 1988, et l'un des rares de Taïwan à avoir engagé un processus d'intégration verticale de ses activités. Celles-ci vont de l'industrie chimique pour la fabrication des fibres synthétiques, à la confection et à la distribution commerciale dans le domaine textile. ${ }^{16}$ Exportatrice vers plus de quarante pays du monde, elle s'est progressivement affirmée comme firme multinationale avec l'implantation de plusieurs entreprises de production en Asie.

La maxime du fondateur de FAR EASTERN «Sincérité, Application, Epargne, Prudence " reste empreinte, dans le choix des termes, de l'idée de communauté fonctionnant, à l'image de la famille, sur le modèle sécuritaire des devoirs réciproques entre le patron et ses employés. Comme dans la plupart des entreprises de la même génération, ce sont les rapports Maître/élèves qui ont prévalu au départ. Et l'Epargne et la Prudence - synonymes d'indépendance financière et de sécurité - ont largement signifié la capacité des dirigeants à assurer la cohésion du groupe autour de la prospérité de l'entreprise.

Mais le sens attribué à ces valeurs cardinales s'inscrit aussi très étroitement dans la situation d'incertitude qui a été celle des entrepreneurs de chine continentale lorsqu'ils sont arrivés à Taïwan, et il reflète avec force l'adhésion de son auteur à la politique du gouvernement nationaliste. Ce qui caractérise en effet FAR EASTERN, c'est l'attachement de la philosophie de son fondateur à un idéal d'affirmation économique, et plus largement politique, qui s'enracine autant dans la tradition commerçante chinoise que dans la conception du développement national prônée, à la suite de SUN Yat-sen, par les dirigeants du Guomindang . Etendre l'assise nationale de la firme, la doter d'une forte autonomie financière pour faire face aux aléas de la conjoncture et assurer son adaptation continue au marché mondial, ont été l'objectif premier des dirigeants.(cf. Doc. en annexe. Far Eastern Group.)

Contrairement au cas de TATUNG, la philosophie de l'entreprise n'est donc pas exclusivement tournée vers l'intérieur, et ce n'est pas tant un modèle d'organisation que la firme aurait à produire pour la société toute entière qui assure à celle-ci son identité : ce qui fait idéal et donne sens aux principes des dirigeants, c'est le fait de symboliser les capacités des entrepreneurs chinois à s'adapter au marché mondial et à renforcer leur développement sur des bases nationales; c'est aussi l'idée que la firme, et plus largement les entrepreneurs chinois qu'elle symbolise, sont porteurs d'une histoire et d'un système de valeurs qui leur confèrent l'aptitude à faire face à la concurrence internationale.

Dans les brochures de présentation de la société, les termes « Sincérité, Application, Epargne, Prudence » sont ainsi toujours rehaussés d'une iconographie abondante et riche en symboles, qui fait constamment référence à l'histoire des échanges entre la Chine et le monde, comme pour exprimer cette volonté d'affirmation économique et de reconnaissance internationale .

41 Imagée de jonques affrontant la mer de Chine, ou de paysages aux reliefs tantôt accidentés, tantôt sereins selon la conjoncture économique à laquelle la firme doit faire face, la route de la soie est sans cesse évoquée pour rappeler les obstacles et les dangers qu'ont eu à affronter les commerçants chinois au cours des siècles, leurs capacités à surmonter une position d'infériorité dans la société chinoise et les freins apportés à leur profession par le pouvoir en Chine. Et ce qui vient en légende ne laisse 
rien ignorer de la capacité de FAR EASTERN à s'adapter à l'économie mondiale: la philosophie de ses dirigeants s'inscrit en droite ligne dans le prolongement de cette histoire : "Traversant le désert, la route de la soie était empruntée par des individus s'efforçant de favoriser un accroissement de l'économie. Dans cet esprit, FAR EASTERN s'est adapté à son environnement économique dans un effort continu pour se positionner avec succès sur le marché international ».

Proche en cela de beaucoup de Chinois d'outre-mer qui ont compensé les discriminations sociales et politiques dont ils étaient l'objet dans leurs pays d'exil en consolidant leur position économique, Y.Z.HSU, le fondateur de FAR EASTERN, a cherché dès le départ à doter son entreprise d'une forte cohésion interne et de solides bases financières.

43 Tout se passe comme si la mémoire des pertes subies en Chine et des entraves apportées au secteur privé par le gouvernement nationaliste avait influencé ses stratégies. La Prudence et l'Epargne semblent avoir signifié pour lui qu'il fallait affranchir la firme d'une dépendance trop forte vis-à-vis du pouvoir politique. Rallié après coup, mais sans aucune ambiguïté au Guomindang, il a d'évidence partagé avec conviction l'idée de relever le défi de 1949, en prouvant par la voie de la "réussite " économique la supériorité du régime nationaliste sur le régime communiste, et peutêtre aussi la chimère d'incarner la Chine entière aux yeux de l'extérieur. Mais si dans le cadre de cette proximité du pouvoir, la firme a bénéficié d'une aide considérable du gouvernement dans les années cinquante et soixante, il semble aussi que ce soit moins en s'appuyant sur leurs relations personnelles qu'en fondant leurs stratégies sur une adhésion à la politique industrielle du gouvernement, que les dirigeants de FAR EASTERN aient tiré avantage de leur engagement au Guomindang : "Nous n'avons pas de dettes politiques», dira son fils ${ }^{17}$; on pourrait ajouter qu'ils ont «joué le jeu du développement national $»^{18}$.

Derrière les termes de Prudence et d'Epargne, ce sont donc à la fois l'idée d'autonomisation vis-à-vis du pouvoir et le souci d'assumer une position sociale et politique à travers une affirmation économique et identitaire de la firme qui sont affiché.

Cette position ambivalente - qui est très représentative de la situation historique particulière dans laquelle se sont trouvés les entrepreneurs de Chine continentale explique en partie que les dirigeants de FAR EASTERN aient placé au cœur de leur logique l'autofinancement des investissements et la notion de risques calculés, tous deux proches de l'idée d'Epargne et de Prudence.

La diversification des investissements, précocement entamée, a toujours été effectuée dans des branches à faibles risques (matériaux de construction, par exemple), ou qui renforçaient l'activité initiale et la position du groupe sur le marché national et international (transports maritimes, distribution commerciale, activités intégrées). FAR EASTERN n'a pas non plus sacrifié au marché international sa position sur le marché national, et le réinvestissement des profits dans la firme a toujours été préféré à une trop forte dispersion des investissements. Si le recours au marché bancaire est désormais très important, c'est toujours 15 à $20 \%$ des profits qui sont ainsi réinvestis en dépenses de capital chaque année. Et FAR EASTERN se distingue de nombreuses firmes taïwanaises par la faculté de ses dirigeants à faire des placements financiers avantageux et adaptés aux situations du moment. 

encore une conception très paternaliste du dirigeant qui sert de référence pour assurer la cohésion des salariés et leur adhésion aux stratégies de la direction ${ }^{20}$. Celui-ci se pose moins comme un autocrate que comme le guide de l'action. Comme au Japon, son "discours est celui du sage qui félicite, qui fait la morale $»^{21}$ et qui indique le sens à prendre pour le bien de tous ceux qui travaillent ou ont investi dans la firme. S'adressant aux actionnaires et aux salariés, Douglas HSU, fils du fondateur, actuellement Président du groupe, termine ainsi son discours de clôture de l'année 1984 par ces mots : "Notre société a fourni de bons résultats ces dernières années... Mais nous devons maintenir notre niveau de compétitivité et suivre les conseils de notre Chairman dans les années à venir ». elle est d'ailleurs rappelée avec force au travers des symboles - dragons, vagues, phoenix - qui ornent les brochures de la société, et qui sont posés là comme pour soutenir les actions nouvelles des dirigeants et renforcer les termes de Sincérité, Application. Tel le dragon - toujours en bonne place - signe de pouvoir, de puissance divine, dont la perle qu'il est censé posséder dans la gorge est «l'éclat indiscutable de la 
parole du chef, la perfection de sa pensée et de ses ordres $»^{22}$ "On ne discute pas la perle $d u$ dragon » disait Mao.

Dans le cas de FAR EASTERN, il s'agit néanmoins d'un paternalisme qui tire sa force principale d'une capacité d'adaptation au monde extérieur. La philosophie de l'entreprise est de ce fait plus " ouverte », toujours chargée de significations nouvelles destinées à soutenir d'autres formes d'organisation et de travail. Plus ou moins mis en avant selon les circonstances, les préceptes d'origine sont ainsi constamment réinterprétés à la lumière de principes de gestion et de modèles stratégiques empruntés aux théoriciens américains de "l'excellence" gestionnaire, dont on comprend aisément qu'ils puissent faire modèle pour les dirigeants de FAR EASTERN.

Influencés par l'expérience japonaise, ces théoriciens semblent en effet avoir "redécouvert les vertus de l'adhésion communautaire», et ils ont largement repris l'idée de communauté soudée autour d'objectifs et de valeurs partagées, plaçant au centre de leur conception du bon fonctionnement de l'entreprise les principes de loyauté, d'honnêteté, d'engagement personnel ou de leadership moral que l'on retrouve dans les entreprises chinoises de Taïwan. ${ }^{23}$

Ce qui leur est emprunté par les dirigeants de FAR EASTERN touche donc essentiellement aux formes de rationalité économique et de gestion modernes qu'ils prônent pour élever la productivité et donner à l'entreprise une plus grande flexibilité.

Ponctuant leurs discours annuels et l'iconographie des brochures, les citations de ces auteurs assurent bien sûr la lisibilité des objectifs de la firme à l'extérieur et sa crédibilité vis-à-vis de ses partenaires étrangers. Mais, dans le choix des termes empruntés, elles ont surtout pour fonction d'enrichir la philosophie de l'entreprise de concepts qui lui donneront un nouveau sens pratique et soutiendront l'idéal d'affirmation économique dans laquelle elle prend corps, et par là d'impulser de nouveaux comportements.

Ce sera tout particulièrement le cas à la fin des années soixante-dix et dans les années quatre-vingt, quand FAR EASTERN - comme l'ensemble de l'industrie taïwanaise - se trouvera confrontée à la récession mondiale et à de nouvelles données de la concurrence internationale, qui placeront la firme en perte de compétitivité sur le marché international: entrée de nouveaux concurrents du Tiers Monde, protectionnisme accru des Etats-Unis et des pays européens et progrès techniques conduisant à une certaine relocalisation de l'industrie textile au Centre, augmentation des coûts de main-d'œuvre, réappréciation du dollar taïwanais, libéralisation des importations à Taïwan... Entre 1984 et 1987, par exemple, les concepts d'Epargne et de Prudence - synonymes de conduite adaptée aux circonstances -, celui d'Application qui veut dire aussi, comme le laisse entendre le terme anglais de Diligence, assiduité à poursuivre un effort soutenu et zèle au travail - sont reformulés à la lumière de deux notions clés : celle d'innovation ou d'esprit d'innovation, conçue " comme une discipline, susceptible d'être apprise et mise en pratique ", qui est empruntée à l'ouvrage de Peter DRUCKER, Innovation and entrepreneurship; celle de reconversion et d'adaptation rapide au changement, nécessitant la définition et la mise en œuvre de nouveaux objectifs à long terme. Et, en 1987, les termes de Sincérité et d'Application sont éclairés par l'idée de responsabilisation et d'esprit d'entreprise à tous les niveaux de la hiérarchie - «chaque salarié, un entrepreneur "- tirée de l'ouvrage de TOM PETERS, A Passion for Excellence. 

succession réussie de Douglas HSU, le fils du fondateur de FAR EASTERN à la tête de la firme - a été poursuivi conjointement à une décentralisation des responsabilités : «innovation très rentable, bien que déviante par rapport à la conception traditionnelle chinoise de l'entreprise qui conduit à conserver le personnel à vie » dira Y.Z. HSU; et une attention particulière a été portée au recrutement de personnel qualifié ayant les compétences requises par les grandes firmes étrangères et souvent formés aux Etats-Unis. Enfin, dans le cadre de l'objectif de diversification qui a été celui des dernières années, la part du textile a été constamment réduite par rapport à d'autres activités (construction, finance, par exemple) et des reconversions engagées dans le domaine industriel. De la production de polyester destinée à l'industrie textile, FAR EASTERN est ainsi passé à la fabrication de bouteilles de plastique et s'est simultanément lancé dans de nouveaux créneaux, telle la production de matériel antipollution, dont le marché est prometteur, notamment à Taïwan où les besoins sont énormes. Les dirigeants ont également cherché à assurer ces nouvelles entreprises d'une forte compétitivité en s'associant à de très grandes firmes, susceptibles de transférer leur technologie, comme Air Liquide par exemple.

61

Portés par une ambition politique certaine, les dirigeants de FAR EASTERN - qui font souvent fonction de diplomates - ont fait de leur firme une ambassadrice de la Chine Nationaliste dans le monde. Et c'est sans doute aussi de cette ambition, qui s'est manifestée par une consolidation simultanée de l'assise nationale et internationale de leurs affaires, que leur philosophie tire en partie sa force.

ACER alias MULTITECH : Porter le progrès technologique au cœur du monde chinois ; être parmi les leaders mondiaux de l'informatique.

62 Enfant terrible de la nouvelle génération des entrepreneurs de Taïwan qui se sont lancés dans la fabrication de micro-ordinateurs au tournant des années quatre-vingt, la firme ACER s'est élevée en moins de quinze ans de simple représentant commercial de marques étrangères au rang de premier producteur et distributeur de microordinateurs de Taïwan. Créée en 1976, avec un capital de départ de 25000 dollars U.S., 
par six jeunes ingénieurs formés à Taïwan, rejoints par la suite par des Chinois venus des Etats-Unis où ils avaient entamé leur vie professionnelle, elle est aujourd'hui à la tête de cinq sociétés employant plus de 3000 personnes et son chiffre d'affaires est estimé à plus de 500 millions de dollars U.S. en $1989^{24}$.

Elle symbolise ainsi tout à la fois la stratégie de reconversion industrielle soutenue dans la dernière décennie par le gouvernement en direction des industries de haute technologie et les capacités de certaines entreprises taïwanaises à se poser progressivement en concurrentes des grandes firmes multinationales dans ce domaine.

De nombreux facteurs, extérieurs à la firme elle-même, mais dont ses fondateurs ont su tirer parti, permettent de comprendre un tel développement ${ }^{25}$; mais pour les dirigeants du groupe ACER, les ressorts essentiels de leur réussite sont moins à chercher du côté de l'environnement économique dont ils ont saisi l'opportunité, que de celui de la conception du travail et des rapports humains qu'ils ont su imposer dans leur entreprise, autrement dit de leur philosophie.

Etre parmi les leaders mondiaux de l'informatique, Porter le progrès technologique au cœur du monde chinois est le double slogan dans lequel celle-ci se trouve ancrée. Associés à des salariés formés dans de grandes firmes américaines, les fondateurs veulent montrer qu'ils ont les capacités de se hisser à leur niveau, ce que le slogan quelque peu provocateur qu'ils adressent à leurs concurrents et partenaires extérieurs ne laisse ignorer : " Nous sommes là et nous y resterons", "Surveillez notre croissance ». Chinois, ils veulent aussi montrer que ceux-ci peuvent acquérir la maitrise des nouvelles technologies, être à l'initiative de leur développement et dépasser le stade de la simple sous-traitance et du piratage.

66 Aussi, dans un domaine où le capital intellectuel, l'innovation et la rapidité de réponse aux changements technologiques sont décisifs, et où paradoxalement l'importance du facteur compétence alimente une forte mobilité du travail qui est déstabilisante pour les entreprises, les dirigeants du groupe ACER - avertis de leur propre expérience d'anciens salariés - ont-ils placés au centre de leur philosophie l'attachement des compétences à l'entreprise, le développement de l'esprit de compétition et de créativité parmi les salariés.

67 Accolés au logo de la firme, leurs « Recettes du Succès " mettent toutes l'accent sur le niveau élevé de qualification et de formation des cadres et employés - « Human Ressources»-, et sur la qualité du travail - «Quality Control»-. Et le professionnalisme, le perfectionnement du niveau des compétences, présentés comme principes intangibles auxquels s'engagent les salariés - «Sincère Commitment "sont là pour assurer à la firme son identité. Quant aux termes de la culture de l'entreprise - «Corporate Culture " - ils sont entièrement consacrés à définir les formes de participation des employés qui soutiendront leur adhésion à ces principes et leur créativité dans le travail. Consignées dans une Charte, ces recettes, qui énoncent de façon un peu fastidieuse les termes du contrat liant les salariés à l'entreprise, se présentent en fait beaucoup moins comme un règlement à suivre que comme un véritable projet partagé, et c'est sans doute ce qui fait leur force de persuasion. Ce qui est imposé aux salariés et ce qu'ils peuvent en recevoir en retour n'est jamais dissocié des performances et objectifs de l'entreprise ; les qualités des dirigeants (persévérance, capacités de travail, dévouement, esprit de compétition...), autrement dit ce qui assure en partie leur réussite, sont constamment prêtées et étendues à l'ensemble des salariés, 
comme vecteur d'une identification permettant de soutenir leur attachement à la firme. (cf. Doc. en annexe. The MSC Group Our Recipe for Success.)

Mélange bien dosé entre le communautarisme chinois et l'égalitarisme à l'américaine, la culture de l'entreprise se structure ainsi autour de deux idées force qui peuvent donner sens à une telle adhésion : "Every employée an entrepreneur ", faire plus à plusieurs, comme au jeu de go, en associant la rapidité tactique du mouvement à l'exécution de la stratégie à plus long terme.

Placé au premier rang des principes, le concept «Innovation through entrepreneurship » donne sens à cette première idée et justifie ce que l'on attend des salariés - «Commitment through perseverence»-, en d'autres termes leur engagement à poursuivre une carrière dans l'entreprise, à s'y dédier, et à partager la responsabilité de son devenir. Comme d'autres entrepreneurs taïwanais, les dirigeants ont donné aux employés la possibilité de participer au capital de l'entreprise, à partir notamment de déductions mensuelles sur leurs salaires - « Employée stock options » - ce qui a permis, du moins au début, de répondre de façon significative aux besoins d'accroissement du capital. Mais contrairement à la plupart de ceux qui ont eu recours à cette épargne interne, ils ne l'ont pas dissociée d'une forte délégation des responsabilités et du pouvoir de décision: ils se sont aussi saisi de la grande homogénéité du personnel en termes d'âge, de formation professionnelle et de motivations, pour étendre à l'ensemble des salariés - notamment les cadres - le fraternalisme d'origine, plaçant les ingénieurs et les techniciens, si ce n'est au même plan, du moins dans un rapport d'égalité avec eux. Ainsi, dans la Charte de l'entreprise, si le patron est présenté comme le symbole de la réussite pour le groupe, ses capacités à diriger la firme - qui sont mises en évidence par ses performances en termes d'âge et de reconnaissance internationale - ne sont pas dissociées des compétences des responsables dirigeants qui sont présentés comme la fierté de tous - «Pride in our top management»-, ni de la qualification et du travail fourni par les autres - «Hard working employées » - qui eux assurent la reconnaissance de l'entreprise - « People make the différence $»$. On retrouve derrière ces termes l'idée de leadership fort et de complémentarité des rôles propres à la tradition chinoise, mais le patron n'est plus l'empereur invisible qui dicte ses lois, il assume pleinement que c'est le partage des compétences et des motivations, la mise en commun de l'ensemble des ressources humaines qui constituent la richesse de l'entreprise.

A travers cette façon de diriger, la participation au capital - devenue «reconnaissance » de la firme envers ses employés - a donc rendu possible l'idée que chacun est un entrepreneur dont l'acte d'entreprendre n'est pas motivé seulement par la rétribution de son travail, mais par l'objectif partagé de faire gagner la compétition que la firme a engagée - «Marathon Relay Spirit ». Les dirigeants précisent euxmêmes que cela a été décisif dans l'engagement des salariés. ${ }^{26}$

71 Dans les faits, cela a en effet permis de soutenir une politique hardie et continue de Recherche/Développement en attachant une main-d'œuvre très qualifiée à l'entreprise. Alors que les salaires des ingénieurs taïwanais sont d'un tiers inférieurs à ceux pratiqués aux Etats-Unis et que le pays doit faire face à une fuite importante des cerveaux, ACER a réussi à recruter 800 chercheurs et ingénieurs entre 1981 et 1986, et à la même date les ingénieurs et techniciens représentaient $60 \%$ du personnel. Accompagnée d'un taux d'investissement dans les activités de recherche représentant $6 \%$ du chiffre d'affaires annuel, cette attraction des compétences, qui s'est exercée non 
pas seulement sur les ingénieurs taïwanais, mais aussi sur un nombre non négligeable de cadres formés aux Etats-Unis - notamment d'origine chinoise, qui ont fait retour à Taïwan - a de toute évidence été un élément décisif du développement de la firme et de ses capacités à élaborer ses propres produits.

Maîtres mots de la culture de l'entreprise, l'Innovation et la Créativité dont a su faire preuve la firme ACER dès sa création, sont d'ailleurs toujours étroitement associés aux ressources humaines et à l'esprit d'équipe qui caractérise le type de gestion adopté « Grow with our Employées » - «Openmindedness in our Management »- et plus généralement à l'idée qu'ensemble, en s'appuyant sur l'initiative de chacun, on peut atteindre cet objectif. Importatrice et sous-traitante de composants pour les grandes marques étrangères, la société d'origine - SERTEK - a été simultanément doublée d'un centre de recherche, et d'une société chargée de la promotion des nouvelles technologies dans le pays et de la formation des ingénieurs à leur utilisation, ce qui, tout en faisant connaître l'entreprise, lui a donné la possibilité de drainer des compétences. Dès 1980, celle-ci crée le premier logiciel en chinois, dont elle développe l'année suivante la production en série et acquiert très vite une position dominante sur les marchés d'Asie pour ce type de produit (plus de $70 \%$ des utilisateurs chinois). En 1981, le premier ordinateur domestique est créé, et fondée la société MULTITECH dont le nom servira de marque aux propres produits de la firme et assurera par la suite la production en série de toute une gamme de compatibles I.B.M. Accusés de piraterie, et ayant à faire face maintenant à l'offensive d'I.B.M. pour se protéger de la production de clones, les dirigeants n'en ont pas moins continué à impulser la création de nouveaux produits permettant à la firme de s'affirmer sur le marché international, changeant même de logo en 1987, pour lui assurer une meilleure image de marque et une plus forte identité sur le marché. En 1988, sa production de micro-ordinateurs atteignait 400000 unités, dont $60 \%$ étaient vendus sous la marque ACER à des prix inférieurs d'un tiers à ceux des grands producteurs mondiaux ${ }^{27}$. Avec un réseau de distribution étendu à 69 pays du monde, ACER n'a cessé d'accroître ses exportations, et, grâce à la qualité de ses produits, a réussi à capter une part importante du marché américain des ordinateurs domestiques.

Mais si la firme s'est montrée innovante et a su faire preuve d'indépendance, c'est aussi que "l'encouragement à la créativité ", " la créativité dans toutes les opérations " , mots clés de la culture de l'entreprise, n'ont jamais été dissociés de l'idée d'adaptation de la production à la demande extérieure - «Market, oriented technology »-. Ils n'ont jamais été dissociés non plus d'une collaboration étroite avec les firmes multinationales pour lesquelles - en raison notamment de l'importance attachée à la qualité ("Total Quality Control»)- ACER a constamment étendu son champ d'activité, tout particulièrement dans le cadre de contrats O.E.M. qui représentent encore aujourd'hui $40 \%$ de sa production.

"Bridging the gap " et "Grow with us " sont les slogans affichés comme objectifs dans la Charte de l'entreprise. Dans les faits, les dirigeants semblent en effet avoir suivi le conseil donné par SUN TZU il y a deux mille ans : "Si l'ennemi laisse la porte ouverte, il faut s'y précipiter $»^{28}$. Au départ ils ont comblé le retard des grandes firmes sur le marché des ordinateurs domestiques et exploité leurs propres possibilités sur le marché chinois. Et dans ce cadre, l'association aux grandes multinationales a de toute évidence constitué le nerf de la guerre, ce qui a permis à la firme de se transférer de nouvelles technologies, d'accroître ses possibilités d'investissement et par là de renforcer son 
potentiel de recherche, ses capacités d'innovation et la compétitivité de ses opérations, mais aussi sa flexibilité et sa rapidité à répondre à de nouvelles demandes sur le marché international.

ACER sort chaque année de nouveaux produits plus performants ; et aujourd'hui, alors que le marché des micro-ordinateurs se montre beaucoup plus concurrentiel - ce qui entraîne des difficultés pour de nombreux fabricants taïwanais, dont ACER qui a été obligé en 1989 de réviser ses prévisions à la baisse - la firme tire de son alliance avec les grandes firmes les capacités de diversifier ses activités dans des domaines plus complexes, qui exigent une forte automatisation de la production et par conséquent de gros investissements. Récemment, ACER a pu augmenter son capital grâce à une participation financière de plusieurs grandes institutions financières, dont la CHASE MANHATTAN BANK, qui y a placé 1,4 millions de dollars. Et ACER s'est associé à TEXAS INSTRUMENTS pour la construction d'une usine de circuits intégrés-mémoires (DRAM : Dynamic Random Access Memories) d'un coût d'environ 250 millions de dollars dont elle a financé l'essentiel, gardant ainsi une certaine indépendance ${ }^{29}$. D'autres alliances seraient envisagées, pour la production de radars. S'appuyant sur la notion de polyvalence affichée dans le logo d'origine, «MULTITECH », les dirigeants cherchent maintenant à diversifier la production dans la fabrication de composants interconnectables sur une multiplicité de systèmes informatiques, de façon à maintenir et accroître leur position sur le marché international. Intermédiaire ou sous-traitant des grandes firmes multinationales? ACER, qui court toujours après les dernières innovations de celles-ci, n'en est pas loin.

Mais comme au jeu de go, qui est rappelé avec force dans les brochures de la société, les dirigeants ont su intégrer à la mise en œuvre de leur stratégie à long terme - Etre parmi les leaders mondiaux de l'informatique - tout un ensemble de mouvements tactiques qui conduisent maintenant à positionner la firme à un niveau mondial. Plutôt que partenaire dépendant, c'est plus comme associée des grandes compagnies, dans un monde devenu de plus en plus interdépendant, qu'elle semble en effet s'affirmer maintenant. Et la philosophie d'ACER, dont une des forces essentielles a été d'attacher les compétences à l'entreprise et d'y développer l'esprit d'innovation à partir d'une forte délégation de responsabilités, mais aussi de faire fonctionner la firme en réseaux interconnectés et ouverts sur l'extérieur, n'y semble pas étrangère.

Tels que les exemples retenus ici permettent de le voir, toutes ces «philosophies » ont pour fonction essentielle d'attacher les salariés à l'entreprise, et de susciter leur adhésion aux objectifs de ses dirigeants. Comme au Japon, ou «sans philosophie, l'action n'est qu'agitation brouillonne $»^{30}$, elles assurent l'ordre, donnent sens au travail, et tendent à gommer les conflits. Dans tous les cas, leur efficience apparaît étroitement liée au caractère fortement polysémique des concepts utilisés dans les maximes qui en condensent l'esprit, polysémie qui permet d'en adapter la signification aux situations changeantes de l'entreprise, et d'en changer le sens selon qu'il s'agit du patron ou de ses employés. Et ce qui en fait aussi la force, c'est l'ancrage des valeurs affichées dans les traditions culturelles et l'histoire de la société, et dans ce cadre, leur attachement à un idéal de vie en société ou d'intégration au monde extérieur qui dépasse le seul cadre de l'entreprise.

Inscrites dans le système de représentation familiale chinois qui place le leader en position de Maître à penser et à agir et lui confère une autorité morale suffisamment forte pour susciter idéal et soumission de la part de ses subordonnés, la loyauté, la 
sincérité, l'application au travail - remplacées plus récemment par les termes d'engagement ou de dévouement - sont autant de valeurs qui ont permis à beaucoup d'entreprises de préserver leur cohésion interne, et par là, d'échapper à l'éclatement qui caractérise un grand nombre d'entreprises chinoises fondées sur des rapports de parenté ou de clans: l'Epargne, valeur proprement paysanne, qui est reprise dans toutes les entreprises, autant pour légitimer de bas salaires, des compressions de personnel ou les investissements engagés, que pour consolider le capital sur des bases internes, a eu aussi pour fonction de renforcer cette cohésion. Et dans sa version moderne, lorsqu'elle est traversée par l'idée de responsabilisation de chacun aux résultats de l'entreprise, cette notion d'épargne semble pouvoir jouer dans le sens d'une plus forte capacité des firmes à se transformer et à se positionner sur le marché mondial.

L'efficience économique de ces philosophies ne vaut néanmoins pleinement, comme le prouvent les exemples retenus, que si l'entrepreneur a les capacités d'en ajuster constamment le sens aux situations dans lesquelles se trouve placée l'entreprise, en y introduisant notamment de nouveaux concepts chargés de faire passer dans la pratique les transformations qu'imposent sa dimension et plus largement son adaptation aux données de la concurrence internationale.

Cette efficience apparait aussi d'autant plus forte que ce qui fait idéal pour l'entreprise se situe du côté du défi économique et que la philosophie prônée par les dirigeants de la firme s'enracine dans une volonté d'affirmation économique et politique vis-à-vis de l'extérieur.

81 Ainsi, bien avant les "apôtres" de l'excellence gestionnaire américains, les entrepreneurs chinois de Taïwan semblent avoir compris qu'une bonne gestion - qui suppose la mobilisation collective - ne peut faire l'impasse du symbolique. Et ce qui fait la force de certains, c'est sans doute de n'avoir pas totalement cédé à cette conception occidentale de la modernité, qui aurait voulu "désenchanter le monde, dissoudre les mythes et abolir les rites $»^{31}$, et de n'en avoir adopté que certaines idées susceptibles de rentabilité économique, en les maintenant bien inscrites dans une éthique partageable par le plus grand nombre.

A Singapour : plus que contrôler et mobiliser : «Implanter dans les esprits » une nouvelle identité

82 La façon dont le gouvernement singapourien matraque et assomme en permanence la population de campagnes idéologiques, politiques et moralisatrices, est indissociable des conditions dans lesquelles il a accédé au pouvoir au moment de l'indépendance tout autant que du projet de société et d'intégration au monde extérieur qu'il a conçu à ce moment-là.

83 Elle est tout aussi inséparable de la conception très autoritaire - pour ne pas dire totalitaire - de l'Etat, que ses dirigeants ont développé dans ce contexte. "Moderne » dans ses modes d'intervention économique, influencé à l'origine par l'idéologie socialdémocrate, le gouvernement singapourien porte aussi tous les stigmates d'un confucianisme que ses dirigeants ont ressuscité pour affirmer leur pouvoir: l'idée d'un Etat tutélaire qui régule les influences extérieures et assure les équilibres internes, d'un Etat fort et vertueux garant d'harmonie sociale et de développement économique, qui tire sa force d'une bureaucratie éclairée très développée, d'un Etat «au-dessus » des hommes et de la société civile qui le constituent. 

irigeants singapouriens, le mot « implanter » revient régulièrement dans les discours officiels. C'est en effet à un changement accéléré de la société que les dirigeants singapouriens ont voulu procéder pour faire passer dans la réalité l'idée d'une ville

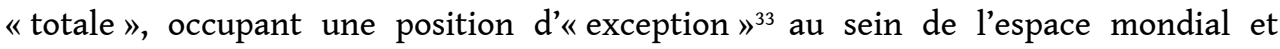
régional. Et, à l'image de leur conception du pouvoir et de cette représentation de la ville - qui n'est pas sans rapport avec leurs origines ${ }^{34}-$, c'est sur la base d'un fort syncrétisme culturel qu'ils ont cherché à donner corps à l'identité singapourienne. S'ils ont fait retour aux valeurs traditionnelles - notamment chinoises - de la société, ils n'en ont en effet repris que ce qui pouvait aller dans le sens de leurs desseins et de la mainmise de l'Etat sur toute la vie économique, politique et sociale, cherchant conjointement à soumettre la population à de nouveaux comportements plus proches de l'Occident. 
conception de la vie en société - importance qui n'a d'égal que le contrôle de tous les moyens d'expression orale et écrite. De cette conception du rôle de l'Etat découle aussi le fait que la propagande officielle touche à tous les aspects de la vie individuelle et collective, et que, très souvent présentée sous la forme de slogans, elle appartienne presque toujours au domaine de l'injonction : « Come on Singapore », «Let's ail do a little bit more »...

91 A exprimer ses idées par des concepts, le gouvernement prendrait-il le risque que le destinataire du message ne se mette à les interpréter à sa façon, puisqu'une idée... « vit avec toutes les autres idées ${ }^{35}$ "? C'est donc surtout sur le mode de l'intimation, du mot d'ordre, que se situent les préceptes du pouvoir. Et ils n'appellent généralement qu'une seule réponse : optempérer - ou déserter. Chargée d'imposer une identité nationale, la propagande officielle, qui participe toujours de l'ambivalence du pouvoir par rapport à la culture, à la langue d'origine, et plus largement à l'histoire, est aussi très pauvre dans ses modes d'expression. Et, à l'image de «la perfection" que prônent les dirigeants pour l'île, l'iconographie qui la soutient toujours très aseptisée et sans vie.

Les injonctions gouvernementales que l'on trouve exposées sur les bâtiments publics, qui sont en anglais - quelquefois traduites dans l'une des autres langues officielles, le chinois, le malais ou le tamoul-, sont ainsi fréquemment redoublées de messages iconiques dont les termes sont souvent ceux de bandes dessinées, comme si le gouvernement s'adressait à un peuple d'enfants. Les personnages qui y figurent sont rarement animés: emblème de Singapour, le lion devient un petit jouet statique bien policé ; seule court l'abeille de la productivité. Et au pays réel, c'est le décor de studio qui est préféré. L'iconographie des affiches qui rythment les campagnes idéologiques du pouvoir semblent ainsi avoir pour fonction d'exorciser la vie, le mouvement. Tout comme les photographies de Singapour, dans les plaquettes des organismes officiels, qui sont systématiquement nettoyées de toute présence humaine, de tout « esprit » du lieu, et présentent la ville comme une gigantesque maquette, plate-forme offshore tout droit sortie des bureaux des technocrates - image à laquelle la ville réelle finit par ressembler.

93 A produire des images plus fortes, le gouvernement s'exposerait-il à voir ressurgir le " tigre ", toujours renaissant, qu'il s'emploie à mater au cœur de chacun?

«COME ON SINGAPORE » ou les recettes du consensus social et politique

Placée au cœur de la devise du gouvernement singapourien «Prosperity, Stability, Social harmony ", la stabilité politique, qui a été considérée à juste titre comme ce qui pouvait permettre à Singapour de devenir un pôle d'attraction du capital international, a toujours été une préoccupation essentielle du pouvoir. Mais une des caractéristiques de celui-ci est certainement d'avoir cherché à atteindre cet objectif en faisant de chaque individu le responsable de la marche d'ensemble de l'économie et en l'impliquant directement dans la mise en œuvre de sa politique et de son projet de société.

95 Coupés de la population peu après avoir accédé au pouvoir, les dirigeants du People Action Party ont tenté d'éliminer toute opposition par la répression. Mais simultanément, ils ont essayé de légitimer leur politique et de se doter d'une base sociale en infiltrant tous les rouages de la société civile. Pour ce faire, ils ont pénétré les organisations communautaires traditionnelles, et les ont supplanté peu à peu, par tout un réseau d'associations contrôlées par le gouvernement, de façon à laisser l'Etat "être le principal agent du changement social $\|^{36}$. 

le pouvoir en contact permanent avec la population. Chargées de toutes sortes de fonctions administratives et d'activités de quartier, voire même de rendre la justice dans les querelles entre voisins, ces associations auront surtout pour fonctions - sous couvert de ces activités - d'informer et de diffuser l'idéologie et la politique gouvernementales, et aussi de capitaliser les informations, les suggestions et griefs éventuels des habitants, pour les récupérer ou les anticiper et étouffer tout conflit ; en bref leur sera dévolue la charge d'assurer un consensus autour de la politique gouvernementale. Mais toujours pour mieux contrôler, mieux intégrer, et empêcher les conflits interethniques, le gouvernement n'a eu de cesse que de chercher à militariser la population, et à l'impliquer dans la défense civile, lui confiant en partie la tâche de maintenir l'ordre et la sécurité urbaine. En 1964, ce sont les Vigilant Corps, formés de volontaires à temps partiel placés sous le contrôle de la police pour surveiller et arrêter les opposants au régime et les fauteurs de troubles, qui sont créés. Puis, dans les années soixante-dix, quand se retirent les forces armées britanniques, l'entraînement militaire prolongé de la population civile devient une arme essentielle du pouvoir pour insuffler l'ordre, la discipline et la soumission au pouvoir : «le développement économique ne peut se produire s'il n'y a pas d'ordre et de sécurité dans ce pays ", dira le Premier Ministre Lee Kuan $\mathrm{Yew}^{37}$. Enfin, dans les années quatre-vingts, lorsque l'opposition tente désespéremment de renaître de ses cendres, et que le maintien de la croissance économique - plusieurs fois ébranlée par les turbulences de l'économie mondiale - nécessite des reconversions importantes, le gouvernement lance une campagne intitulée "Total Défense", destinée à mobiliser la population sur tous les fronts de la politique gouvernementale, sous couvert des menaces - notamment extérieures - qui pèseraient sur le devenir de la Ville-Etat. Les Vigilant Corps sont alors remplacés par de nouveaux organes de surveillance rapprochée dans les grands ensembles d'habitation, organisés en Comités de Résidents dépendants de la Défense, dont le rôle est d'assurer la sécurité. Simultanément les habitants sont appelés à faire respecter la loi, par le biais notamment de la délation, qui est alors encouragée publiquement par l'Etat.

Véritables milices politiques, ces organes de défense civile ont eu au départ pour fonction essentielle de mener la répression. Mais, plus largement, c'est l'idée de faire de chacun le défenseur de la loi et le responsable de son application pour lier dans les faits la population au pouvoir qui a été affirmée

dans cette pratique. Comme sur le panneau indiquant la proximité d'un poste de police de voisinage "Neighbourhood police post", qui figure deux citoyens enlacés à un homme en uniforme, où se lit le souci de faire de la société une communauté entièrement soudée au pouvoir. Dans l'iconographie que celui-ci produit, c'est toujours comme si le retrait d'un seul pouvait menacer la société dans son ensemble. Et la phrase d'un volontaire définissant le sens qu'il donne à l'une des campagnes gouvernementales de défense civile ne fait que renvoyer en miroir cette représentation de la société : «Cela nous aide à être plus proches les uns des autres » - et laisserait penser que l'objectif est atteint.

Dans le droit fil de cela, la moralisation de la société a été une constante de l'action gouvernementale: pour imposer à chacun - jusque dans sa vie privée - des façons d'être qui correspondent à l'image et aux fonctions qu'entend donner à Singapour le gouvernement et renforcer le sentiment d'appartenance nationale; pour créer une uniformité de comportement qui permettrait autant de gommer les différences 
"raciales ${ }^{38}$ que d'affirmer « l'exemplarité » de la société singapourienne vis-à-vis de l'extérieur; enfin pour placer la population dans une logique d'effort et de sacrifice qui la mette en position d'adhérer sans broncher à la politique du pouvoir. (cf. Doc. en annexe. Come on Singapore.)

On retrouve en partie ceci dans le Code de Bonne Conduite destiné aux membres des Comités de Vigilants. Ce qui y est souligné, ce ne sont pas seulement les fonctions qu'ils ont à remplir, mais les valeurs de loyauté, de dévouement à la tâche, de travail ou d'exemplarité vis-à-vis des autres, ce qu'ils doivent « être » en tant que citoyens, mais aussi en tant que voisins et individus - le gouvernement allant même jusqu'à dicter les règles d'autocontrôlé du corps qui devraient être, dans son esprit, celles de tout bon Singapourien.

101 C'est aussi ce que laisse entendre une des affiches de la campagne entreprise autour du thème de la courtoisie en 1985 : «It's so nice to be courteous "- traduit pour la circonstance en malais et en tamoul -: quelle que soit la culture ou la langue, tous pareils, tous rassemblés dans les mêmes H.L.M. derrière le Lion. Image bien pauvre et raccourcie d'une nation... Mais "quand les emblèmes furent inventés, les démons s'enfuirent en gémissant ${ }^{39}$. Emblème de Singapour, le Lion fait ainsi figure de fauve bien dompté, comme pour signifier le refoulement de l'opposition et du conflit.

La stabilité politique assurée, restait à mobiliser la population sur le plan économique et à la soumettre aux impératifs contenus dans la stratégie de développement adoptée pour maintenir la Ville-Etat en position de relais privilégié du capital international. Fort de la surveillance rapprochée de la population, le gouvernement a ainsi constamment accompagné sa politique économique d'une propagande abondante en direction des travailleurs, pour soutenir la productivité du travail, et - élément essentiel de l'attrait des investissements étrangers - empêcher tout conflit dans les entreprises.

103 Telle par exemple la campagne menée en 1985 sur le thème de la " Productivité ", à un moment où les tentatives du gouvernement d'assurer une reconversion de l'industrie vers les hautes technologies ne semblait pas avoir produit tous les effets attendus, et où celle-ci se trouvait soumise à une concurrence plus forte de la part des pays voisins. Dans la façon d'aborder le problème, "Together we'll succeed ", on retrouve l'idée que l'implication de chacun est essentielle ; celle-ci est même présentée comme vitale, et la «Prospérité ", premier terme de la devise du gouvernement, est posée, sur le mode de l'injonction, comme liée à l'addition et à la conjonction d'un ensemble d'activités et d'efforts individuels : "Each of us » «Let's all do a little bit more ». (cf. Doc. en annexe. Productivity begins at home. Come on Singapore.)

104 «Ensemble » est le mot clé. Qu'il s'agisse de la ruche ou de la famille, il vient comme en écho de la "complémentarité » confucéenne, selon laquelle l'être humain ne peut exister qu'en se fondant dans la société et doit concourir à assurer l'ordre et l'harmonie. Ce qui définit cette harmonie, c'est que chacun soit à sa place et y excelle. ${ }^{40}$

105 Si les fonctions sociales sont ainsi distinguées, dans la ruche productiviste toutes les abeilles sont semblables, asexuées, sans différences ethniques - hormis un vague turban - : c'est à nouveau l'homogénéité, la totalité - synonymes ici de cohésion - qui sont affirmées. Pour former le $\mathrm{S}$ de Singapour, les abeilles font la ronde, ou la chaîne, elles se tiennent, accrochées aux couvre-chefs militaires que portent celles situées en haut de l'image. 
Mais là aussi, dans l'esprit des dirigeants, seule l'inculcation d'une morale mettant en avant "effort, épargne, sacrifice " et sentiment d'appartenance communautaire pouvait semble-t-il assurer une telle mobilisation au travail et satisfaire aux exigences de la cohésion nationale. A partir des années soixante-dix, retour fut donc fait aux valeurs et aux langues d'origine, précédemment évacuées au profit de l'anglais, et d'une façon toujours plus affirmée par la suite, le pouvoir n'a eu de cesse alors que de renforcer l'éducation morale de la population. Il affiche de plus en plus sa hantise que la ville ne soit contaminée par les idées de liberté et de démocratisation, et par l'individualisme, vu comme une cause de déclin occidental face au Japon, fort de ses valeurs communautaires. L'exacerbation de l'individualisme, dans cette société d'immigrants où la seule religion fut longtemps le "moneytheism $\|^{41}$ et la détérioration morale, le relâchement au travail, le manque d'engagement social qui l'accompagnent lui apparaissent comme autant de menaces virtuelles pour le succès du pays. Mais il veut aussi affirmer Singapour comme ville chinoise, prouver la supériorité de la culture chinoise et de ses pratiques politiques, notamment par rapport à celles de ses voisins. Les valeurs essentielles d'une éthique nationale chargée de légitimer le fait que, pour être riches en Asie de l'Est, il convient de ne pas être libres, seront ainsi principalement puisées dans le confucianisme. Et, de la morale et des traditions de la population, ne seront reprises que les valeurs susceptibles de renforcer le "communautarisme ", en d'autres termes la responsabilité sociale et la conscience civique, la soumission aux impératifs d'une économie dépendante obligée de s'adapter rapidement aux changements extérieurs : cohésion et harmonie familiale, piété filiale, pragmatisme, vengeance, autant de valeurs issues de la Chine ancienne, constamment exaltées à la télévision par les séries historiques et dramatiques en mandarin retraçant des scènes de vie chinoise qui sont importées à cet effet.

Aux écoles reviendra en premier lieu la charge d'enseigner aux enfants les concepts moraux qui doivent définir ou particulariser la société et assurer la cohésion nationale. 42

Mais c'est surtout dans la famille - rempart contre l'individualisme - qui sera pour les dirigeants la matrice de toutes les communautés, que doit s'effectuer

l'apprentissage de la discipline, l'inculcation de la morale de l'effort pour chacun, le respect du pouvoir : ainsi, pour Lee Kuan Yew, c'est l'observation de deux principes de base qui assurerait la survie à long terme de Singapour: "la famille est l'unité de base de la société ", " l'individu est moins que la société ". ${ }^{43} \mathrm{Et}$ la cohésion familiale, la piété filiale, consolidées dans les faits par une politique de logement incitant à la cohabitation des générations, subordonnant les femmes à des mesures démographiques, voire eugéniques, et limitant leur accès à certaines fonctions, ont été des ressorts essentiels de l'action gouvernementale. ${ }^{44}$

110 "Productivity begins at home" titre l'affiche de la campagne destinée à engager le pays à mieux résister à la concurrence internationale; et l'iconographie qui appuie cette idée, autant que ce qui l'accompagne en légende - «By adopting a positive attitude, we can achieve great thingsfor our Nation»- ne laisse pas ignorer les intentions gouvernementales. Avant de passer à table, de consommer "la prospérité », tout le monde, homme, femme, enfants, est aux tâches ménagères et semble travailler avec plaisir, dans l'harmonie la plus parfaite d'une famille "moderne» mononucléaire, image inventée pour la circonstance comme pour souligner l'idée de modernité contenue dans le concept de Productivité. «Harmonie sociale »-synonyme dans les 
faits de consensus social et politique dans la soumission - tel est un des trois principes clés du gouvernement singapourien...

De là, la représentation figée, saisie d'immobilité qu'il donne de la société au travers de l'affiche célébrant le vingt-cinquième anniversaire de "l'indépendance». Dans l'impossibilité où il s'est placé de faire référence aux temps fondateurs, à la lutte pour l'indépendance nationale, rien ne renvoie dans cette image à l'histoire. Et, s'il y a forclusion de l'histoire, les « principes » du gouvernement qui devraient faire idéal sont même noyés en petits caractères dans le texte d'accompagnement. L'efficience propre à la langue chinoise s'est perdue. Du symbolique au spéculaire : là où la polysémie des concepts pourrait donner une liberté de penser, une créativité, ce qui triomphe ici c'est une image à laquelle chacun est censé trouver à coller la sienne.

112 Le message iconique est simple : en travaillant ensemble et en suivant la musique, on décroche le trophée. Sur l'affiche tous chantent, mais un seul joue, véritable metteur en chœur, maître de cérémonie, parmi ces douze femmes et treize hommes, censés figurer les vingt-cinq ans fêtés.

113 Tout est orchestré par l'Etat, destin politique, stratégies économiques, vie sociale, tout le monde mobilisé, intégré. L'Etat donne le la, la référence à suivre. Et «quand la Musique est parfaite, il n'y a plus de rébellion, quand les Rites sont parfaits, il n'y a plus de querelle $»{ }^{45}$

114 Les vingt-cinq Singapouriens, privés de leur mémoire, sont comme " arrêtés ", tous ont l'air revêtus de panoplies. Seules citations des cultures traditionnelles, un ou deux costumes, comme déplacés, parmi ces citoyens blanchis, et un vieux qui tend... sa cage. Tous les enfants sont chinois (alors que dans la réalité, au grand dam du pouvoir, le taux de reproduction des Chinois est très inférieur à celui des Malais et des Indiens). Et tout en haut de l'affiche, l'armée veille : «Stand up Singapore ». L'harmonie est celle des uniformes...

A regarder ces affiches, on se souvient de ce que Barthes écrivait du mythe : «Les choses perdent en lui le souvenir de leur fabrication (...) On a fait disparaitre toute trace salissante d'origine ou de choix (...) La fin même des mythes, c'est d'immobiliser le monde ».46

Sans doute la mobilisation incessante de la population a-t-elle été entendue : la mise au travail, de façon efficace et disciplinée, de l'ensemble de la population a été réussie, et les multinationales continuent à préférer Singapour aux pays voisins. Mais le moralisme et le dirigisme suscitent aussi des freins au développement : la productivité augmente moins rapidement que dans d'autres nouveaux pays industriels d'Asie, et le gouvernement arrive difficilement à juguler l'exil des cerveaux et le manque de maind'œuvre qualifiée. A vouloir, au nom de la Paix sociale et de la compétitivité économique, imposer à la population des normes étroites de pensée, à force de chercher à uniformiser la société, c'est à une certaine stérilité et à une certaine désespérance qu'aboutit aussi ce régime : alors que les jeunes diplômés n'excellent pas par leur créativité, ce qui entrave la reconversion de l'industrie vers des activités de plus haute technologie, le taux de suicide, dans cette ville si bien policée, est inquiétant. L'analyse de toutes ces épigraphes donne ainsi à lire des différences très sensibles entre les deux pays, quant aux modes d'exercice du pouvoir politique et à ses rapports à la culture et au symbolique.

118 A Singapour, l'Etat garde le monopole du pouvoir symbolique. Du contrôle et de l'utilisation abusive de l'espace graphique - instrument majeur de leurs « utopies » - les 
dirigeants qui l'incarnent ont cherché à acquérir les forces de créer de toutes pièces une ville " offshore " qui ne ressemble à aucune autre et à conforter l'image d'un Etat «vertueux » exerçant son pouvoir pour le bien de tous. Comme le disait Confucius, « Le Prince n'a pas besoin de tuer ", sa vertu " est le vent qui courbe l'herbe ». ${ }^{47}$

Forts d'une étatisation très importante de l'économie, qui s'inscrit dans cette conception du pouvoir, et qui s'est trouvée favorisée par la politique d'ouverture de l'île aux multinationales et par le développement d'une bourgeoisie commerçante et financière peu ancrée dans le territoire national, ils restent les Maîtres du jeu sur tous les fronts. Et, alors que le vent de la démocratisation et des libertés individuelles souffle sur un grand nombre de régimes autoritaires, l'opposition, qui tend à se manifester de nouveau, ne semble pas encore capable de résister à la répression politique et culturelle que le gouvernement impose à la société.

Dans cette ville ouverte à toutes les circulations marchandes ou financières et à toutes les compétences techniques, où tout est fait pour limiter la circulation des idées, l'espace urbain - entièrement remodelé par l'Etat - affiche un contraste étonnant entre une grande richesse matérielle et un certain dénuement culturel: pas de place sur les murs pour les inscriptions sauvages, une presse muselée et contrôlée par l'Etat, peu de lieux de sociabilité ouverts sur l'extérieur. Et dans les librairies où foisonnent les ouvrages de gestion, d'informatique, de cuisine ou de jardinage, les rayons de littérature, d'histoire et de sciences humaines restent dégarnis.

A Taiwan, les rapports entre l'Etat et la société civile n'ont pas été du même ordre et leur évolution récente a été dans le sens de la démocratisation. De type dictatorial, le régime s'est appuyé jusqu'à ces dernières années sur une très forte répression politique et sur une propagande très active des "Principes » de SUN Yat-sen dont se réclame le Guomindang, qui ont servi de lignes directrices aux stratégies gouvernementales depuis 1949. Conjointement à l'embrigadement de la population dans les rangs du parti nationaliste, ces Principes sont ainsi rappelles avec vigueur dans tous les discours et publications du gouvernement, ou au travers des médias et jusque dans les chambres d'hôtel, où ils figurent au même titre que la Bible. Mais la censure des moyens d'expression s'est essentiellement cantonnée au domaine politique, notamment aux productions critiques à l'égard du régime, ou qui faisaient référence à la Chine Populaire. Et, hormis l'inculcation de ces principes, et le fait de toujours exhiber aux frontons des bâtiments officiels la carte de la Chine, le gouvernement nationaliste ne s'est pas montré aussi prolixe en images et en préceptes qu'à Singapour, et ne s'est pas arrogé le monopole du pouvoir symbolique.

Plutôt que de renforcer l'étatisation de l'économie - le dirigisme lui a été préféré - il a surtout encouragé la formation d'une large couche d'entrepreneurs industriels, ayant de fortes ramifications dans l'ensemble du territoire national. Et il leur a en partie délégué la charge d'encadrer la population - voire de lui dispenser un enseignement moral et politique - puis celle de représenter le régime dans l'arène internationale. Aussi la marge de manœuvre laissée à la société civile a-t-elle été plus importante, laissant place progressivement à son émergence sur le plan politique et à un processus de démocratisation.

Pour des raisons politiques évidentes, la valorisation de la culture et de l'histoire chinoise a été une des lignes directrices du pouvoir et la production intellectuelle bien que longtemps très contrôlée - a été encouragée. Alors que $23 \%$ de la population 
a poursuivi des études post-secondaires, l'exode des cerveaux se ralentit et, fort d'une main d'œuvre qualifiée, Taïwan semble pouvoir se lancer dans les hautes technologies.

D'un régime autoritaire à l'autre! Lorsque l'on passe de Singapour à Taipeh, on ne manque pas d'être étonné : les espaces de sociabilité - cafés, maisons de thé à la japonaise... - y sont nombreux, les librairies aussi - Taïwan est un des plus grands pirates mondiaux en matière d'édition. Et l'univers des rues, peuplées d'une population très vivante, est rempli de signes, de graphismes colorés et de nombreuses boutiques où se vendent pinceaux, encres, papiers ou matériels de calligraphie...

\section{NOTES}

1. Marcel GRANET - 1968 - La pensée chinoise, Albin Michel, Paris.

2. Marcel GRANET, op. cit.

3. Selon l'expression de B. KARLGREN, cité par ETIEMBLE in Ecriture, Encyclopaedia Universalis, 1985.

4. Contrairement à ce qu'il en est en Chine Populaire, où les idéogrammes ont été très simplifiés à dater de 1954, c'est toujours l'écriture traditionnelle qui est enseignée à Taïwan.

5. Cf. photo offerte par SUN FA-MIN, à l'issue de l'entretien qu'il nous a accordé à Taipeh en décembre 1986.

6. Selon l'expression d'Armando PETRUCCI, in «Storia e Geografia délie culture scritte », cité par Roger CHARTIER, Le Monde Liber n 1, octobre 1989

au XIXe siècle", in Les racines de l'entreprise, Revue Française de Gestion, 70, oct. 1988.

7. Sur la question générale des rapports entre gestion et culture, on se reportera à l'ouvrage de Philippe d'IRIBARNE - 1989 - La logique de l'honneur. Gestion des entreprises et traditions nationales, Le Seuil, Paris.

8. Cette pratique n'est évidemment pas spécifique aux entrepreneurs chinois, bien qu'elle soit au centre de leur conception du « leadership ». A la fin du siècle dernier, elle était ainsi très fréquente dans les entreprises familiales françaises, où le développement d'œuvres sociales et d'écoles ouvrières était pour le patronat un des moyens utilisés pour acquérir une main d'œuvre qualifiée, et surtout la « fidéliser » à l'entreprise. Comme chez beaucoup d'entrepreneurs taïwanais, l'autofinancement et le réinvestissement des profits étaient aussi au cœur de la logique patrimoniale du patronat français. Voir à ce sujet: Daniel HENRI, « Capitalisme familial et gestion industrielle au XIX ${ }^{e}$ siècle ", in « Les racines de l'entreprise ", Revue Française de Gestion, 70, oct. 1988.

9. Nous faisons ici référence aux théoriciens américains de la gestion qui, dans le sillage de T. PETERS et de son ouvrage In search of Excellence, ont pris la mesure de l'importance des rapports humains et de l'implication des salariés dans la marche des entreprises. Ces auteurs, dont la réflexion résulte en partie de la prise de conscience des performances des entreprises japonaises, ont abordé la question centrale des rapports entre gestion et culture, et cherché à définir l'ensemble des valeurs morales et des 
conduites qui pouvaient assurer plus d'efficience au travail, une plus grande flexibilité et une plus forte créativité. Cf. Philippe d'IRIBARNE, op. cit.

10. Lorsque nous parlons des entrepreneurs originaires de Chine continentale, nous faisons référence à ceux qui se sont installés à Taïwan en 1949 et après ; nous désignons par « entrepreneurs taïwanais » les natifs de l'île.

11. Entretien réalisé avec T.S. LIN à Taipeh en décembre 1986.

12. Il semble se situer là dans le droit fil de la pensée nationaliste chinoise : pour SUN Yat-sen, en effet, la question essentielle, dans une société où les intérêts de la famille, pendant des millénaires, ont primé sur tous les autres et limité la constitution de solidarités plus larges, était d'arriver à transférer les valeurs familiales et claniques dans d'autres cadres, à dépasser l'antagonisme entre groupes de parenté traditionnels et nation « moderne ». Dans cette perspective, ce qui est recherché, plutôt qu'une individualisation croissante comme en Occident, c'est l'ancrage dans le sol des liens traditionnels - d'une façon un peu similaire à ce qui a si souvent été décrit pour le Japon -.

13. Le taux d'épargne y atteint $40 \%$.

14. Cf. Sixième Convention nationale du Guomindang en Chine en 1945 : "profit-sharing should be set up in industrial enterprises". (Cité par T.K.DJANG in Industry and Labor in Taiwan, Monograph Séries 10, Academia Sinica, Taipei, 1977, p. 285). Cette position a été réaffirmée plusieurs fois par la suite.

15. OEM : original-equipment manufacturer. Il s'agit de contrats de sous-traitance dans le cadre desquels les produits fabriqués par l'entreprise sous-traitante sont conformes à ceux des grandes firmes étrangères pour lesquelles elle travaille, mais sont vendus sur le marché sous d'autres marques que les leurs.

16. C'est sur FAR EASTERN TEXTILE CORPORATION, principale firme du groupe, que nous centrons notre analyse.

17. Entretien avec Douglas HSU réalisée à Taipeh en décembre 1986.

18. Lorsque par exemple dans les années soixante, la production textile de Taïwan est menacée par le protectionnisme des Etats-Unis à l'égard de certains produits, les dirigeants de FAR EASTERN ont certainement bénéficié, comme d'autres continentaux, d'une part très importante des quotas à l'exportation pour soutenir son activité. Mais très vite, ils ont engagé d'importants investissements dans la production des fibres synthétiques, pour maintenir et étendre la position de la firme sur les marchés extérieurs, soutenant ainsi la politique gouvernementale qui visait à moderniser l'industrie textile et, plus généralement, à consolider l'industrie en assurant une remontée de filière. Et lorsqu'ils ont eu à se heurter à la politique très restrictive du gouvernement en matière de financement, c'est vers le marché « parallèle » qu'ils se sont tournés pour soutenir l'expansion de la firme.

19. Mot japonais dont l'usage s'est répandu internationalement pour désigner les devises des entreprises.

20. Paternalisme marqué, comme dans d'autres firmes taïwanaises, du souci de jouer un rôle dans la société. FAR EASTERN a ainsi développé de nombreuses organisations à vocation sociale : une fondation pour la promotion des activités culturelles et scientifiques, un hôpital, une fondation médicale, un institut de technologie qui compte 3000 étudiants et, plus récemment, un collège technique destiné à former 4500 élèves par an.

21. Bernard GALAMBAUD - 1988 - Spiritualité sans bavure,. Autrement $n^{\circ} 100$. « Le Culte de l'entreprise ». 
22. Jean CHEVALIER, Alain GHEERBRANT - 1982 - Dictionnaire des symboles, Robert Laffont, Paris.

23. Philippe d'IRIBARNE, op. cit.

24. FAR EASTERN ECONOMIC REVIEW, 2/11/1989.

25. L'ascension rapide de ACER est en effet indissociable du concours de circonstances externes et internes qui l'ont rendue possible : le retard pris par les grands producteurs mondiaux comme I.B.M. dans la fabrication de micro-ordinateurs à un moment d'expansion extrêmement rapide du marché domestique, et l'intérêt des grandes marques à créer leur propre concurrence interne par l'intermédiaire de contrats O.E.M. assurant la diffusion de clones à prix moins élevés ; l'ouverture du marché taïwanais à l'informatique ; l'existence à Taïwan d'un important vivier d'ingénieurs et un écart de salaire très favorable pour le pays ; enfin les soutiens apportés par le gouvernement taïwanais à ces nouvelles industries et à l'attraction des compétences chinoises développées à l'extérieur, notamment aux Etats-Unis.

26. Entretien avec John WANG, Vice-Président du MSC GROUP, Taipeh, décembre 1986. 27. FAR EASTERN ECONOMIC REVIEW, 17/8/1989.

28. SUN TZU, Les règles de l'art militaire, cité par D. PY in « Sun Tzu au Forum des Halles ", Le Monde, 25.11.1989.

29. FAR EASTERN ECONOMIC REVIEW, 14/1/1988, 17/8/1989 et 2/11/1989.

30. Bernard GALAMBAUD, op. cit.

31. Philippe d'IRIBARNE, op. cit. p. 259.

32. Lee Kun Yew, 7/9/1968, cité par Jean-Louis Margolin, 1989, Singapour, 1959-1987, Genèse d'un nouveau pays industriel, L'Harmattan, Paris.

33. Cf. Charles GOLDBLUM, 1983, « Singapour, l'Etat d'exception », Hérodote. «L'implosion urbaine ».

34. Descendants des Chinois installés de longue date dans les Détroits, les dirigeants singapouriens de la première génération - issus pour certains de familles ayant servi dans l'administration coloniale -, n'étaient pas tous de Singapour, et déjà très occidentalisés. Sans appartenances précises, intellectuels formés pour la plupart en Grande-Bretagne, nombre d'entre eux avaient même perdu l'usage de la langue chinoise.

35. Jacques LACAN - 1975 - Les écrits techniques de Freud. Séminaire 1, Le Seuil, Paris.

36. CHANG HENG SHEE - 1976 - The dynamic of one party dominance ; the P.A.P. at the grass roots. Singapore University Press.

37. Sur ce sujet, se reporter à J.L. MARGOLIN, op. cit.

38. Terminologie fréquente du gouvernement pour exprimer les différences ethniques et culturelles.

39. 39 Marcel GRANET, op. cit., p 47.

40. "Chaque être a une place, tout objet un usage ; tout est là où il se doit (...) Que le coq veille sur la nuit, que le chat attrape les rats, chacun a son emploi et le maître est sans émoi ». Exposé doctrinal de Han Fei, cité par Jean LEVI in Les fonctionnaires divins, Le Seuil, Paris, 1989, p. 134.

41. Selon l'expression du ministre des Affaires étrangères RAJARATNAM en 1973.

Cf. FAR EASTERN ECONOMIC REVIEW du 1/8/1980.

42. Cf. Any religion is useful to good government, FEER, 1/8/1980, et CHANG HENG CHEE in Le Monde du 20/6/1983. Voir aussi J.L. MARGOLIN, « Singapour. Bureaucratie, confucianisme, libéralisme », in Bureaucraties chinoises, Textes rassemblés par R. LEW et 
F. THIERRY, L'Harmattan Asie-Débat 3, 1986, p. 151-181 et « Comment peut-on être Singapourien » in Espaces Temps 42, 1989.

43. Cf. FEER du 9/2/1989. Voir aussi les FEER du 26/2/1982 et du 7/5/1982.

44. Cf. FEER du 31/8/1989.

45. Li Ki, cité par Marcel GRANET, op. cit., p 336.

46. Roland BARTHES - 1957 - « Le mythe aujourd'hui » in Mythologies, Seuil.

47. CONFUCIUS, Les entretiens familiers (XII, 18), cité par ETIEMBLE in Confucius, Gallimard, 1986.

\section{RÉSUMÉS}

Aujourd'hui, le recours au symbolique, et tout particulièrement aux mots écrits, semble garder une place majeure dans l'art de gouverner en pays chinois et on ne peut manquer d'être frappé par l'importance que lui accordent ceux qui détiennent le pouvoir économique ou politique à Taïwan et à Singapour. L'analyse de quelques maximes d'entrepreneurs de Taïwan, celle de prescriptions politiques du gouvernement singapourien, constituent autant d'éléments qui aident à comprendre les conceptions et pratiques du pouvoir dans ces pays.

Graphie spaces and leadership practices : entrepreneurial maxims in Taiwan, political precepts in Singapore

In Chinese countries, nowadays, symbolic and mainly written words seem to be keeping a major rôle in the art of goveming. It is particularly noticeable in Taiwan and Singapore that political and business leaders pay spécial attention to it. The analysis of some entrepreneurial maxims in Taiwan as well as political directives of the Singaporean government, contributes to the understanding of leadership conceptions and practices in thèse countries.

\section{INDEX}

Mots-clés : Singapour, Nouveaux Pays Industriels, espace graphique, pouvoir, culture d'entreprise, entrepreneurs

Keywords : Singapore, New Industrial Countries, Taiwan, graphie spaces, leadership, entreprise philosophy, enttcpreneurs

\section{AUTEURS}

\section{CATHERINE PAIX}

Chargée de recherches au CNRS, elle travaille actuellement sur les stratégies des principaux acteurs du développement de plusieurs N.P.I. d'Asie - les entrepreneurs et la classe politique -. Elle prépare dans ce cadre un ouvrage collectif sur les « bourgeoisies » du Tiers Monde. 


\section{MICHÈLE PETIT}

Ingénieur au CNRS, elle est rattachée au Laboratoire STRATES. Elle poursuit actuellement des recherches sur les entrepreneurs de plusieurs pays d'Asie (Taiwan, Singapour, Hong Kong). 